This document is confidential and is proprietary to the American Chemical Society and its authors. Do not copy or disclose without written permission. If you have received this item in error, notify the sender and delete all copies.

\title{
Amphiphilic peptide self-assembly: Expansion to hybrid materials
}

\begin{tabular}{|r|l|}
\hline Journal: & Biomacromolecules \\
\hline Manuscript ID & bm-2017-00764t.R2 \\
\hline Manuscript Type: & Review \\
\hline Date Submitted by the Author: & 03-Aug-2017 \\
\hline Complete List of Authors: & $\begin{array}{l}\text { Mikhalevich, Viktoria; University of Basel, Department of Chemistry } \\
\text { Craciun, Ioana; Universitat Basel Departement Chemie } \\
\text { Kyropoulou, Myrto; Universitat Basel Departement Chemie } \\
\text { Palivan, Cornelia; University of Basel, Chemistry Department } \\
\text { Meier, Wolfgang; University of Basel, Department of Chemistry }\end{array}$ \\
\hline
\end{tabular}

\section{SCHOLARONE \\ Manuscripts}




\title{
Amphiphilic peptide self-assembly: Expansion to
}

\author{
hybrid materials
}

Viktoria Mikhalevich †, Ioana Craciun †े, Myrto Kyropoulou $\dagger$, Cornelia G. Palivan †, Wolfgang

Meier * $^{*}$

$\dagger$ University of Basel, Department of Chemistry, Klingelbergstrasse 80, 4056, Basel, Switzerland

\begin{abstract}
The design of functional systems with sizes in the nanometer range is a key challenge in fields such as biomedicine, nanotechnology or engineering. Some of the most promising materials nowadays consist of self-assembling peptides or peptide-polymer hybrid materials because of the versatility, and the resulting properties, which can be achieved with these structures. Selfassembly of pure amphiphilic peptides or in combination with block copolymers results in a large variety of nanostructures (micelles, nanoparticles (NPs), compartments, planar membranes) each with different characteristics and tunable properties. Here, we describe such novel peptideor peptide-polymer-based supramolecular nanostructures, and emphasize their functionality and various promising applications.
\end{abstract}

Keywords: vesicles, membranes, peptide-polymer conjugates, peptide nanostructure 


\section{Introduction}

Peptides possess unique properties, such as bioactivity and good biocompatibility, and serve as the major molecular assemblies and scaffolds in nature at the nano-scale, micro-scale, and macro-scale. ${ }^{1}$ There, the peptide building blocks serve as scaffolds for different bio-assemblies including collagen, keratin, pearl and actin. ${ }^{2}$ Although there are only 20 natural amino acids, the variety of combinations results in an amazing diversity in material properties and functionalities. The self-assembly process is mediated through non-covalent interactions including hydrogen bonding, van der Waals, electrostatic, and stacking interactions. ${ }^{3}$ Certain thermodynamic and kinetic conditions are leading to the self-assembly of peptides. These mechanisms can be explained, for example by multiscale molecular simulations, showing dynamics of hierarchical self-assembly from the molecular to mesoscale level. ${ }^{4}$ The ability of the peptides to form specific secondary structures helps to design nanomaterials with controllable structural features. Taking advantage of intrinsic properties of natural peptides, a huge number of peptide-based building blocks, such as cyclic peptides (CPs), amphiphilic peptides, copolypeptides, surfactant-like oligopeptides, dendritic peptides, and aromatic dipeptides, have been developed for different supramolecular structures, and their possible applications in biology and nanotechnology are explored. ${ }^{5,6-7}$ Their key advantages include biocompatibility, biodegradability, and flexibility. Particularly appealing are the amphiphilic peptides because they can self-assemble into supramolecular structures, such as nanotubes (NTs), NPs, vesicles, and micelles, depending on their chemical and physical properties (sequence, charge, size etc). ${ }^{8-11}$ Such supramolecular 
assemblies have been proposed for carrier-mediated drug delivery, tissue engineering, biomineralization, molecular imaging and membrane protein stabilization. ${ }^{12,8,13-14}$

The sequence of amphiphilic peptides is organized in two regions-the hydrophobic and the hydrophilic part. The hydrophilic part is usually composed of amino acids with hydrophilic side chains, such as lysine or glutamic acid, whilst the hydrophobic part can consist of amino acids with non-polar side chains, such as tryptophan or valine. ${ }^{15}$ A special case are the so called "peptide amphiphiles", in which the hydrophobic part consists of an alkyl chain attached to a biofunctional peptide epitope. ${ }^{16}$ These conjugates are able to self-assemble into high-aspect-ratio cylindrical nanofibers upon triggering via light, $\mathrm{pH}$, ions, and enzymes. ${ }^{17}$

Another way to expand on the potential of peptide-based structures is by using a combination of peptides and synthetic polymers to obtain functional hybrid materials. The combination between these two families of compounds is a useful tool, where the selective and yet diverse nature of peptides is enhanced by the mechanical stability and robustness of synthetic polymers. In order for these novel hybrid materials to reach their full potential, the functionality of the peptides should remain unaltered during the polymer-peptide assembly. If this requirement is fulfilled, then new opportunities for applications in various fields open up. In this review, we will focus on some important nanostructures of self-assembled peptides and peptide/polymer hybrid systems. We will also emphasize different properties and applications, such as biomedical or filtration membranes.

2. Self-assembled amphiphilic peptide nanostructures: NPs, micelles and vesicles Self-assembling peptides are synthesized via solid-phase peptide synthesis, or recombinantly produced in bacteria. The sequence of amino acids that make up the peptide, as well as the 
choice of self-assembling process, all together determine the types of nanostructures that can be obtained. $^{11,}$ 18-19 Peptides rely on non-covalent binding interactions between amino acid sequences to form ordered secondary structures, such as $\beta$-sheets, where the peptide chains are aligned laterally and held in place by back bone hydrogen bonding interactions between the chains, and $\alpha$-helices, where the peptide chain adopts a spiral conformation again held in place by back bone hydrogen bonding interactions along the peptide sequence. Self-assembly of peptides into various nanostructures is promoted by designing amphiphilic peptides or by incorporating aromatic amino acids such as tryptophan and phenylalanine that can form noncovalent binding interaction via $\pi$-stacking. ${ }^{20-21}$ These aromatic amino acid sequences can be further coupled to hydrophilic amino acids to afford self-assembling amphiphilic peptides. In this section, we focus on recent developments and applications of self-assembled amphiphilic peptide NPs, micelles and vesicles.

\section{Self-assembled peptide NPS}

Self-assembled spherical peptide NPs, if smaller than $100 \mathrm{~nm}$ in diameter, are particularly amenable for biomedical applications because they are readily uptaken by cells. NPs can load hydrophobic drugs within their inner core during the self-assembly process thus making them ideal candidates for drug delivery systems. ${ }^{22-23}$ One such self-assembling peptide is EAK16-II (amino acid sequence: n-AEAEAKAKAEAEAKAK-c) that formed NPs of approximately 100 $\mathrm{nm}$ in diameter and successfully encapsulated a hydrophobic anticancer agent ellipticine. ${ }^{24}$ These small NPs were readily uptaken by A549 lung carcinoma cells and exhibited high cytotoxicity. Also, by changing the peptide to hydrophobic drug ratio, NPs in the micron range were obtained, however due to their large size they had a significantly lower uptake and related cytotoxicity. 
Such NP systems can be designed to be responsive to various biologically relevant stimuli such as $\mathrm{pH}$, redox potential, metal ions or even specific enzymes resulting in release of the encapsulated cargo. ${ }^{25-27}$

Peptide sequences that form helical coiled-coil motifs can also be self-assembled into peptide NPs. In one such example, a peptide that forms two helical coiled-coil motifs, with a short linker region in between the two helices, was meticulously studied to determine the effect of point mutations in the linker region on the self-assembling process. ${ }^{28}$ Studies that use in silico modeling of peptide sequences combined with in vitro testing to optimize peptide length, and the overall self-assembly into small peptide NPs, aid in the global understanding of the peptide selfassembling process.

Antimicrobial peptides, most often containing sequences of positively charged arginine and lysine residues, have been self-assembled into peptide NPs as well. ${ }^{29-30}$ Antimicrobial peptides can be highly toxic towards not only bacteria, but also normal cells; however, self-assembling into various nanostructures such as NPs, can result in more selective peptides because the selfassembling process alters the charge distribution and secondary structure of the peptide. ${ }^{31}$ A major issue still surrounding such peptides is their susceptibility to inactivation by proteases. One way of overcoming protease degradation can be achieved by tethering the antimicrobial peptides to a substrate such as gold NPs. ${ }^{32}$ It was shown that the antimicrobial peptides maintain their activity and have increased stability towards trypsin digestion. Depending on the selfassembling properties of the peptide tethered to the gold NP, they can further assemble into NP superstructures. $^{33}$

Self-assembled peptide micelles 
Amphiphilic peptides can also self-assemble to form micelles that contain a hydrophobic inner core and hydrophilic exterior corona. Peptide amphiphiles that contain a charged head group conjugated to a long hydrophobic tail were self-assembled to form micelles. ${ }^{30,34}$ Such micelles, similarly to NPs, have been explored for applications as drug delivery systems for hydrophobic drugs or imaging agents, and have been shown to have low toxicity in vivo and can be cleared rapidly through the renal system. ${ }^{35}$

Apart from longer peptide amphiphiles, peptides containing aromatic residues, such as truncated gramicidin A (gA) sequence composed of L-tryptophan-D-leucine repeating units, self-assemble into micellar structures. These peptides can be coupled via a reduction sensitive linker to a hydrophilic histidine rich domain to obtain stimuli-responsive NPs (Figure 1). ${ }^{36}$ The amphiphilic peptides first assembled into micelles, entrapping doxorubicin (DOX), an anticancer drug, within the hydrophobic domain and antisense oligonucleotides along their hydrophilic outer core. Secondly, the micelles self-assembled into spherical NPs, termed peptide beads, of 100-200 nm. ${ }^{33,37}$ These peptide beads can not only deliver both hydrophobic and hydrophilic payloads but can also selectively release them upon a physiological trigger, such as the reductive environment surrounding tumors.

Peptide micelles can also be decorated with functional peptide motifs, targeting ligands as well as epitopes to the ends of the peptide thus providing selectivity for specific cell types. ${ }^{38}$ By incorporating these motifs onto self-assembling peptides, their affinity for select targets is enhanced and their application can be expanded. Thermally responsive elastin-like peptides have been conjugated to tumor targeting recombinant Llama heavy-chain antibody fragments (VHHs) against epidermal growth factor receptor (EGFR). ${ }^{39}$ Alongside the targeting VHHs', a photosensitizer was also linked to the elastin-like peptide. When heated, the elastin-like peptides 
assembled into micelles exposing both the targeting VHHs and the photosensitizer. Upon illumination, cells expressing EGFR are selectively killed. This example illustrates the versatility that can be obtained through the use of self-assembling peptides to obtain both stimuliresponsive as well as target selective nanostructures.

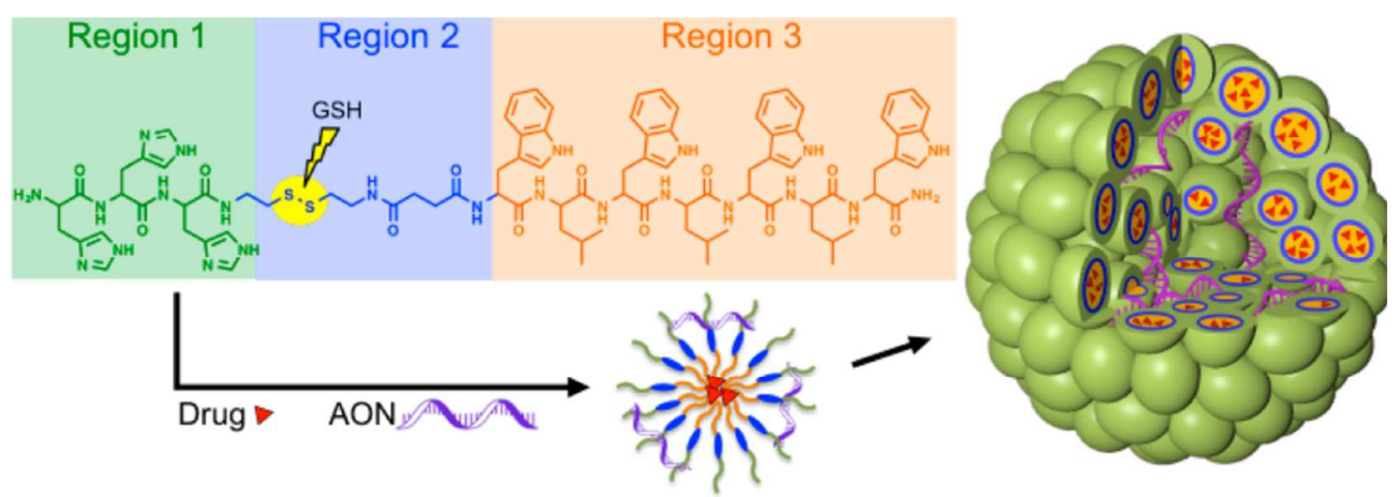

Figure 1. Self-assembly of amphiphilic peptides containing a histidine rich hydrophilic domain (region 1) connected by reduction sensitive linker (region 2) to a tryptophan rich hydrophobic domain (region 3). A two-step self-assembly can occur: first, the peptides assemble into micelles loaded with DOX within the inner hydrophobic core and a nucleotide in the hydrophilic exterior; secondly, the micelles cluster together to form multicompartment micelles. Reprinted from ref. 36. Copyright 2016 American Chemical Society. ${ }^{29}$ Originally adapted from ref. 40 with permission of The Royal Society of Chemistry. http://dx.doi.org/10.1039/c4bm00230j ${ }^{40}$

\section{Self-assembled peptide vesicles}

Apart from NPs and micelles, amphiphilic peptides can self-assemble into vesicular structures as well. Peptide vesicles are versatile candidates for designing drug delivery systems, as active molecules can be encapsulated within the inner core of the vesicles or inserted in their membrane. ${ }^{41}$ One approach to generate peptide vesicles is by using lipid-like peptides where the peptide chain contains a charged head group connected to a long hydrophobic tail. The charged 
head group can be either positively charged via incorporation of positively charged lysine residues or negatively charged via the incorporation of negatively charged aspartic acid residues. The hydrophobic tail contains non-polar residues such as repeating alanine residues. In a recently published study, such peptides were employed as sustained release drug delivery systems. ${ }^{42}$ Another approach to form vesicular assemblies is based on short water-soluble dipeptides. An interesting architecture obtained consists of a multicompartment architecture in which small peptide vesicles are encapsulated within a larger one. ${ }^{43}$ These multicompartment structures were shown to be $\mathrm{pH}$ stable but could disassemble in the presence of calcium ions releasing both an encapsulated dye and cyclic adenosine monophosphate. While these mutivesicular structures were $\mathrm{pH}$ stable, other peptide vesicles are designed to assemble and disassemble based on changes in $\mathrm{pH}$. For instance, recombinant peptides containing glutamic acid residues that form a hydrophilic domain followed by a sequence of alanine, valine and leucine residues that comprise the hydrophobic domain of the oligopeptide, were shown to self-assemble into vesicles at $\mathrm{pH}$ values above $5 .^{44}$ At $\mathrm{pH} 4$ these vesicles disassembled and formed aggregates, however neutralizing the $\mathrm{pH}$ reversed this process and the peptides once again reassembled into vesicles. Peptide-based drugs have gained increased momentum in the past couple of years, with more and more hitting the market and even more undergoing clinical trials. While still in the developmental stage, self-assembled peptide NPs, micelles and vesicles have shown great promise for applications as drug delivery systems, nanoscaffolds for tissue engineering, and diagnostic probes. ${ }^{9,} 45$ Preparing large quantities of peptides can be quite expensive and obtaining the desired final nanostructure is not always guaranteed when working with synthetic peptides. One way to improve upon these systems is to incorporate self-assembling peptides into polymeric structures, thus obtaining more stable and multifunctional hybrid materials. 
3. Peptide-Polymer conjugation: synthesis and applications

Hybrid materials based on the combination of peptides with copolymers takes advantage of the polymer science that is able to provide well-controlled structures, down to the molecular level, and multiple chemical functionalities. ${ }^{46}$ Polymers have exceptional properties regarding stability, they are able to exhibit stimuli responsiveness and some of them, amphiphilic copolymers, are able to self-assemble in different nano-structures and mimic biological materials. One of the most important features of such amphiphilic block copolymers is the ability to self-assemble into mono- and bilayers. The bilayer membranes can appear in different shapes, such as planar or three-dimensional vesicular structures. ${ }^{47-48}$ Diblock copolymers (AB), symmetric triblock copolymers $(\mathrm{ABA})$ or asymmetric triblock copolymers $(\mathrm{ABC})$ are used to self-assemble into a large variety of supramolecular assemblies. Note that usually when we discuss about amphiphilic polymers $\mathrm{A}$ and $\mathrm{C}$ stand for the hydrophilic domains, and B for the hydrophobic domain.

The chemical conjugation of peptides to polymers, or as commonly called the functionalization of polymers with peptides, leading to the self-assembly of various nanostructures takes place mainly via a specific type of chemical reaction. ${ }^{49}$ The so-called "click" reaction is the most common way to achieve the conjugation of a peptide to a synthetic polymer. ${ }^{50}$ The most important parameters for these reactions are: i) a high yield ii) mild beginning conditions (aqueous solution, room temperature, physiologic $\mathrm{pH}$ ), and iii) small amounts or byproducts. Apart from the classic approach, which is the use of Copper-catalyzed azide-alkyne cycloaddition, many other types of "click" reactions have been reported, as for example strainpromoted azide-alkyne cycloaddition, thiol-ene reaction, Diels-Alder reaction, oxime ligation. ${ }^{51}$ Depending on specific "click" chemistry, the reaction conditions for peptide-polymer conjugates 
vary accordingly. The main bio-applications of peptide-polymer conjugates that self-assemble into supramolecular assemblies, are drug and gene delivery systems, and tissue regeneration. ${ }^{52}$ A recent example of a nano-sized drug-delivery system is based on the conjugation of angiopep2 peptide with poly(dimethylsiloxane)-poly(2-methyloxazoline) (PDMS-PMOXA) diblock copolymer via 1-ethyl-3-(3-dimethylaminopropyl)-carbodiimide/N-hydroxysuccinimide coupling chemistry (Figure 2). ${ }^{53}$ Angiopep2, is a targeting peptide with 19 amino acids with high binding affinity to the low density lipoprotein receptor-related protein 1 and therefore with the ability to pass the Blood Brain Barrier and target glioma cells. The angiopeg2 functionalized polymer formed polymer vesicles (polymersomes), which served as drug nanocarriers, when loaded with DOX. ${ }^{54}$ The presence of angiopep2 peptide triggered the cellar uptake of the DOXloaded polymersomes, which selectively released DOX into glioblastoma U87MG cells in vitro. The polymer membrane (thickness of $16 \mathrm{~nm}$ ) protected the drug, allowing it to diffuse slowly through.

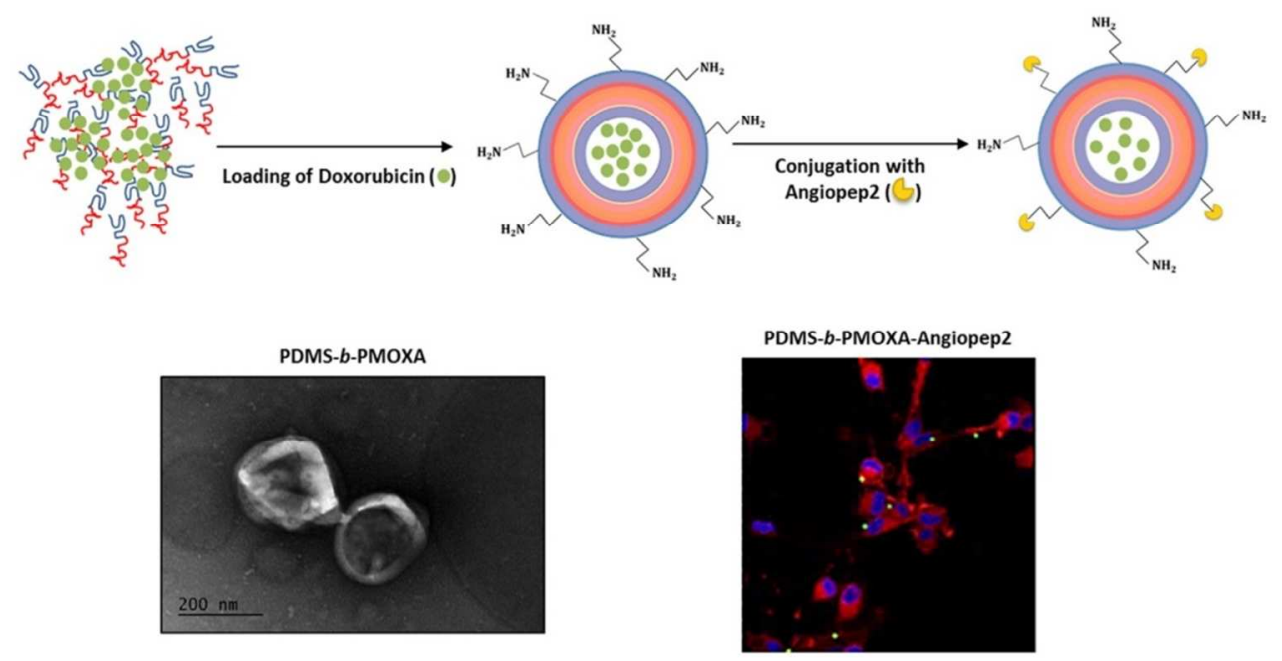

Figure 2. Encapsulation of DOX into peptide-polymer vesicles, functionalized with peptide Angiopep2. Reprinted from ref. 53, with permission from Elsevier. 
In another example, polymersomes have been functionalized with peptides for high binding specificity to colon cancer cells. ${ }^{55}$ Polymersomes based on block copolymer poly(ethylene oxide)-b-poly(1,2-butadiene) were externally functionalized, via an azide-alkyne "click" reaction with a fibronectin mimetic peptide PR_b (with sequence KSSPHSRN(SG) $)_{5}$ RGDSP). This conjugation not only promoted the cell uptake, but also led to a controlled delivery of an antitumor drug into colon cancer cells in vitro. A similar concept has been exploited when polystyrene-block-poly[L-isocyanoalanine(2-thiophen-3-yl-ethyl) amide] based polymersomes were externally functionalized with cell penetrating peptides $(\mathrm{CCPs}) .{ }^{56} \mathrm{CCPs}$-functionalized polymersomes loaded with either green fluorescent protein or horseradish peroxidase were up taken by HeLa cells, and served to release in vitro the encapsulated proteins.

Apart from the conjugation of polymers and peptides, the insertion of peptide-based ion-channels into polymer supramolecular structures represents another strategy to combine, in a functional manner, peptides and polymers. Alamethicin was used to control the ion concentration within copolymer giant vesicles during mineralization of calcium phosphate, ${ }^{57}$ whilst the insertion of $\alpha$ HL into PB-PEO vesicles induced calcein leakage. ${ }^{58}$ The functional insertion of gA inside the membrane of poly(2-methyloxazoline)-block-poly-(dimethysiloxane)-block-poly(2methyloxazoline) PMOXA-PDMS-PMOXA polymersomes served to obtain stimuli-responsive polymersomes that preserve their architecture, whilst their membrane has stimuli-responsive permeability. gA is a short helical polypeptide from Bacillus brevis that forms a transmembrane channel when inserted in lipidic or polymer membranes, and allows the passage of small cations (including $\left.\mathrm{H}^{+}\right) .{ }^{59}$ Indeed, successful insertion of gA did not affect the stability and architecture of the polymersomes, and resulted in a rapid influx of protons and monovalent ions through the 
membrane (Figure $3 \mathrm{~A}-\mathrm{C}) .{ }^{60}$ The change of the fluorescence intensity of 5(6)-carboxyfluorescein encapsulated inside polymersomes after gA insertion, served to prove the functional insertion of these biopores in synthetic membranes that are significantly thicker (thicknesses of the membrane ranging from 9.2 to $16.2 \mathrm{~nm}$ ) than the size of the gA (Figure $3 \mathrm{D}, \mathrm{E}$ ). $\mathrm{gA}$ has been inserted and remained functional in membranes with a thicknesses of $\leq 13.1 \mathrm{~nm}$, which is more than 4 times higher than the size of gA. This unexpected result is due to the high flexibility of the synthetic membranes based on PMOXA-PDMS-PMOXA, which overcome the mismatch between the pore length and the membrane thickness.

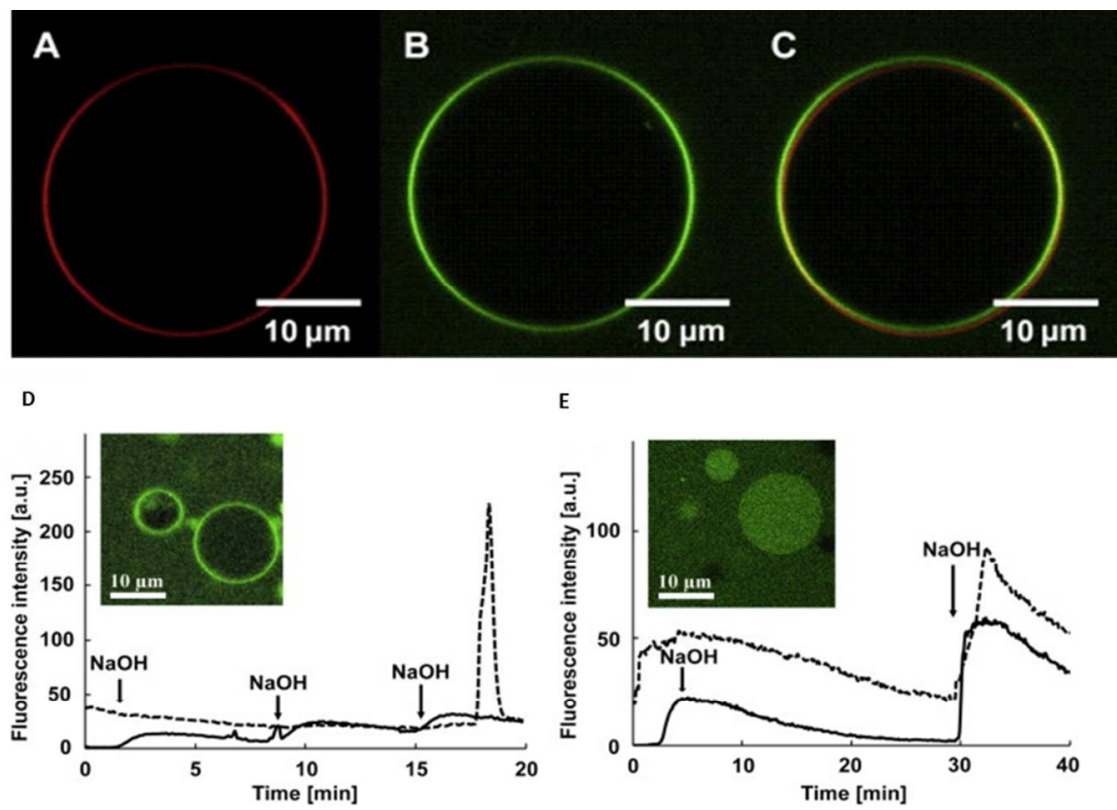

Figure 3. CLSM images of a giant unilamellar vesicle (GUV) composed of $A_{7} B_{49} A_{7}$ triblock copolymer in the presence of: (A) Bodipy (red), (B) labeled gA-OG488 (green), (C) Overlay of A and B (red and green). Fluorescent intensity change over time of 5(6)-carboxyfluorescein inside and outside of polymer GUVs (D) in absence and (E) after insertion of gA. Reprinted from ref. 60, with permission from Elsevier. 
Another type of polymer-peptide hybrid system concerns NPs based on amphiphilic block copolymers which are grafted with peptides. ${ }^{61}$ In particular, proline-valine-glycine-leucineisoleucine-glycine peptides were conjugated to a biodegradable polymer, poly(trimethylene carbonate) via UV activated "click" thiol-ene chemistry. Such NPs have potential for biomedical applications in targeted cancer therapy because the peptides can be cleaved selectively by the well-known tumor-associated enzyme matrix metalloproteinases 2 and 9 (MMP-2 and MMP-9) that appear in very high concentration in aggressive tumors. Similarly, degradable and biocompatible poly(benzyl malate) NPs decorated with biotinylated cyclic RGD peptides, and loaded with the anticancer drug DOX, were efficiently up taken by HepaRG cells. ${ }^{62}$ In another example, PMOXA was combined with a cleavable peptide block poly(aspartic acid) (PASP), and finally modified with diethylenetriamine (DET). PMOXA-b-PASP(DET) self-assembled into biodegradable and biocompatible NPs after complexation with plasmid DNA (pDNA), and served to efficiently transfect HEK293 and HeLa cells with green fluorescent protein (GFP) pDNA in vitro. ${ }^{63}$

A different approach to form polymer-peptide materials is based on the integration of CPs within polymer NTs. ${ }^{64}$ Eight- amino acid based CPs act as precursors for the polymerization of the polymer N-isopropylacrylamide. The peptide-polymer material self-assembles into hybrid NTs, constructed by a peptide core and a covalently attached polymeric coating. This creates a novel class of non-toxic hybrid NTs with promising application in biomedical technology. In another example, CPs were used in combination with several polymers including poly(butyl acrylate), poly(dimethyl amino ethyl acrylate), poly(acrylic acid), poly(styrene) and poly(hydroxyl ethyl acrylate) for efficient and controlled preparation of peptide-polymer NTs. ${ }^{65}$ Later on, a formation of a peptide- polymer NT was reported (Figure 4). ${ }^{66}$ In this work, both thiol-terminated polymers 
and alkyne-terminated polymers where conjugated with cyclic oligopeptides generating polymerpeptide NTs with potential application as synthetic transmembrane protein channel mimics. The novelty in these particular NTs is that they offer the possibility to self-assemble into either Janus or mixed corona form. They also performed a Calcein dye leakage experiment using large unilamellar vesicles (LUVs) where no leakage of the dye was observed for the conjugates.

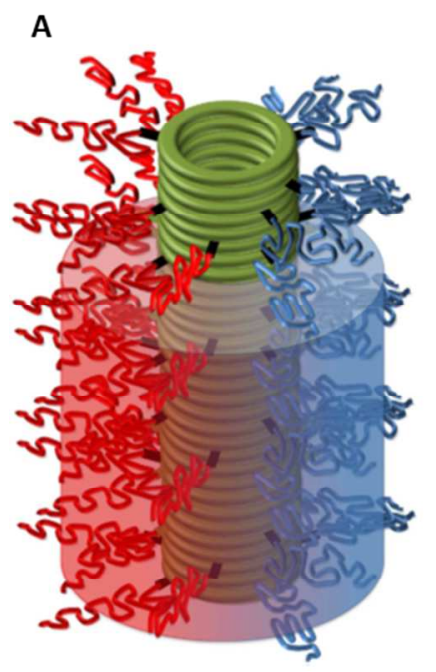

B

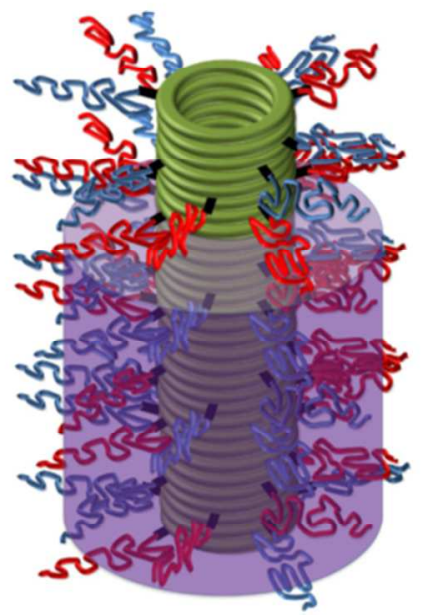

C

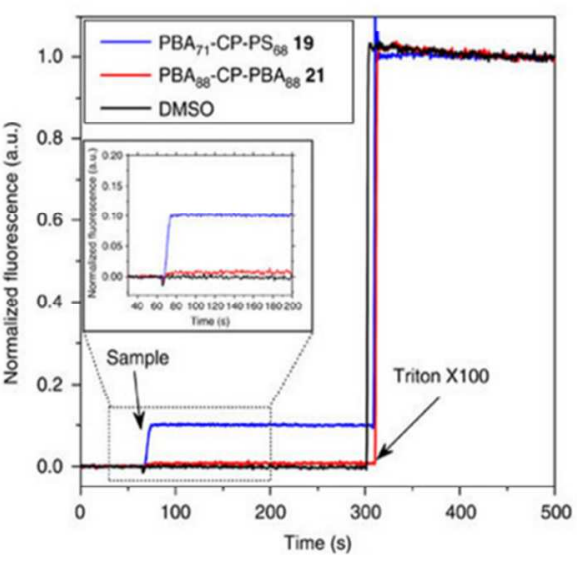

Figure 4. CP-polymer NTs with two corona configurations: (A) Janus assembly with 'demixed' corona. (B) Hybrid assembly with 'mixed' corona (C) Calcein dye leakage experiment using LUVs with $2 \mu \mathrm{M}$ Janus conjugate. No dye leakage was observed for non-Janus conjugate. Reprinted by permission from Macmillan Publishers Ltd: Nature Communications, ref. 66, Copyright 2013. https://www.nature.com/articles/ncomms3780

The exploitation of polymer-peptide self-assembly processes which leads to self-organized supramolecular structures nanostructures (polymersomes, NTs, NPs) opens fascinating and 
promising routes in biotechnology, nanotechnology and nanomedicine. At the same time, polymer-peptide conjugates dilate upon more complex structures, such as polymer films, but the functional reconstitution of peptide structures into planar polymer membranes remains highly challenging and there are many parameters yet to be investigated and defined, as the peptides do not appear any more in their natural environment.

\section{Reconstitution of peptides in planar polymer membranes}

Another strategy to generate peptide-polymer hybrid materials is to integrate peptides into solid supported or free standing planar polymer membranes, which have high potential for applications as bio-sensing platforms. In this respect, pore formation in polymer membranes is in focus by now, due to the advantage of the membrane technology for separation, and more precisely for the transport selectivity of the membrane. ${ }^{67}$ Various peptides, such as gA, alamethicin, or melittin are able to form pores in cell membranes, lipid membranes, but also in copolymer membranes. ${ }^{68-70}$ The phase behavior of alamethicin, an antimicrobiotic peptide, in planar Langmuir polymer films on the air-water interface was extensively studied in the group of Meier, to gain a deep insight into their physicochemical properties. ${ }^{71}$ Films of PMOXA-PDMSPMOXA triblock copolymers with different lengths were used to insert alamethicin. The mixing properties of this copolymer with peptides resulting on mixed monolayers and the phase behavior were dependent on the length of the copolymer. The monolayer at the air-water interface was compressed and showed progression of the domain growth and their changes in shape (Figure 5). The reason for the changes was that the larger polymer had a greater flexibility and ability to adopt more conformations, and therefore more possibilities to host the peptide. 
Additionally, at high surface pressures, the polymer matrix stabilized the peptide and preserved it from collapse.
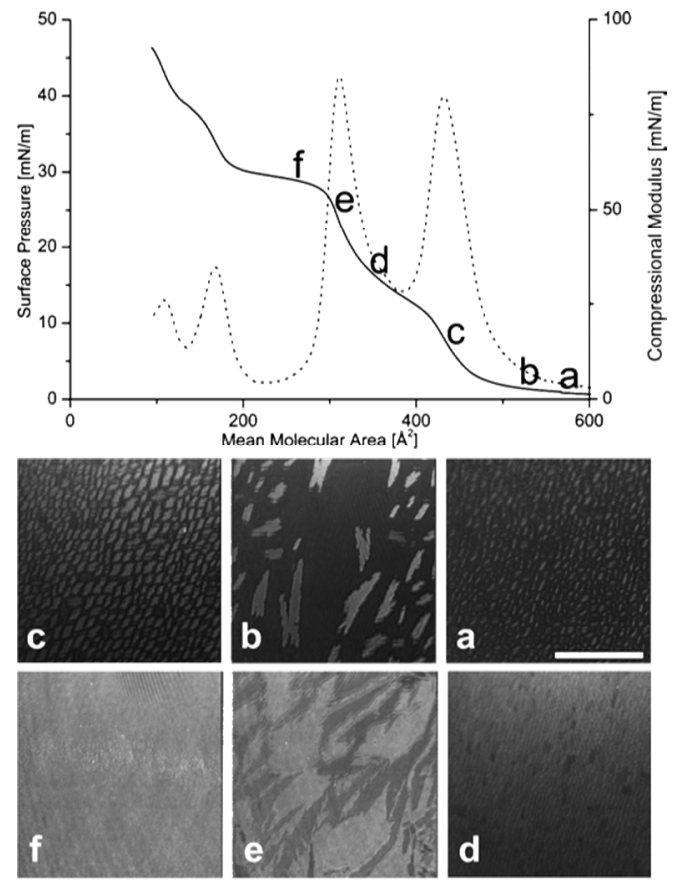

Figure 5. BAM images of a $\mathrm{PMOXA}_{13} \mathrm{PDMS}_{23} \mathrm{PMOXA}_{13}$-alamethicin (molar ratio 0.3:0.7) monolayer at different surface pressures. Reprinted by permission from ref. 71. Copyright 2006 American Chemical Society.

The effect of the polymer environment on the function of incorporated peptides (for example $\alpha$ hemolysin ( $\alpha$-HL) and alamethicin) was first described using a single molecule transport pore in free-standing membranes (9 and $5.7 \mathrm{~nm}$ lengths). ${ }^{72}$ Unfortunately, in contrast to alamethicin, $\alpha \mathrm{HL}$ was not able to be incorporated into the thick polymer membrane. Interesting, the measured functionality of the incorporated peptides (e.g. conductance) was very similar to their behavior in conventional lipid membranes. When gA peptide was added to polymer membranes, it was able to adopt distinct conformations in different environments: inside membrane arrays made of 
PMOXA-PDMS-PMOXA it preserved its functionality indicating a high potential for highthroughput screening applications. ${ }^{70}$ In another approach, planar membranes with synthetic AB and ABA block copolymers of PMOXA and PDMS were used to study the reconstitution of alamethicin and gA and compared with their reconstitution in lipid bilayers. ${ }^{73}$ Alamethicin was successfully inserted and showed gating properties similar to those found in diphytanoyl phosphocholine lipid bilayers, whilst gA behavior in polymer membranes was different then in lipid bilayers: the conductance was significantly lower, and there was no evidence of conductance-state switching.

An amazing breakthrough in the field was the functional pore formation of peptides in solidsupported planar membranes showed by the Meier group. The functionality of a peptide pore in a solid-supported polymer membrane has been established with $\alpha$-HL as model. ${ }^{74} \alpha-\mathrm{HL}$ was functionally incorporated in an artificial polymer, tethered, solid-supported bilayer membrane (TSSBM) based on poly(butadiene) -block-poly(ethylene oxide) (Figure $6 \mathrm{~A}){ }^{75}$ The copolymer was transferred to a surface by using the so called Langmuir-Blodgett and Langmuir-Schaefer transfer technique, which allows control of layer density by the surface pressure of the molecules. After a surfactant-free insertion of $\alpha-\mathrm{HL}$, the electrical conductance across the membrane indicated that the peptide was functionally inserted and the ion flow through was modeled with a Donan potential (Figure 6 B). Such peptide-TSSBMs can be used for drug screening, trace analyzing, and biosensing. 

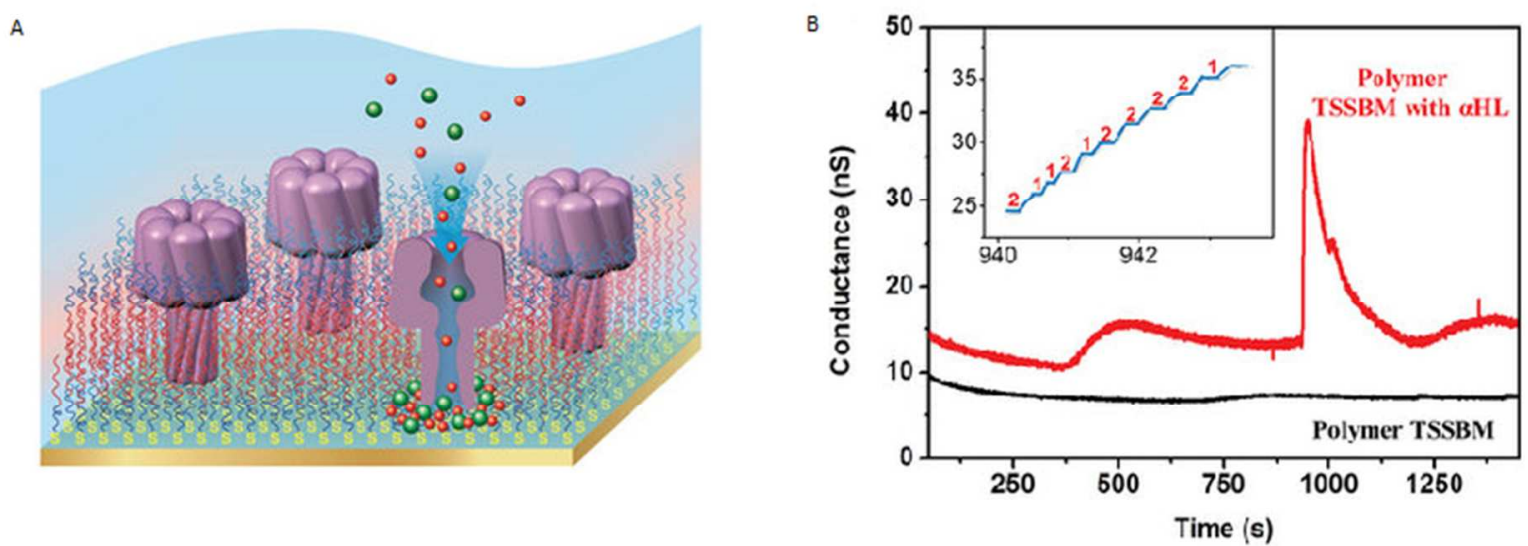

Figure 6. Schematic representation of the TSSBM (A) and conductance measurement across it (B). Reprinted by permission from Macmillan Publishers Ltd: Scientific Reports, ref. 75, Copyright 2013. https://www.nature.com/articles/srep02196

The usage of peptides for pore formation in polymer membranes becomes more and more important, because in contrast to the insertion of membrane proteins, no difficult expression and no surfactants are needed for peptides. The field of self-assembled polymer structures with peptides incorporated hold promising applications in the area of highly selective separations, high-throughput screenings or biosensing.

\section{Conclusions and future perspectives}

Peptidic supramolecular assemblies with sizes in the nanometer range have gained traction in recent years as biomaterials due to the versatility of peptides themselves to self-assemble into various architectures for the design of complex systems, as well as recent advancements in peptide synthesis which enables further research and design of non-naturally occurring peptides. Peptides that can self-assemble into supramolecular architectures such as micelles, nanofibrils, 
NTs or vesicles, are of particular interest firstly due to the innate properties of the peptide building blocks regarding biocompatibility and roles in multiple biological systems and secondly, the gained advantages such supramolecular architectures possess, for example stability with retained functionality.

Peptide vesicles and NPs have been shown to be of use in medical applications as drug delivery or imaging tools as they can be loaded with active compounds (drugs, proteins, DNA) or fluorescent probes and selectively accumulate at specific bio-locations, as for example at tumor sites through the enhanced permeability and retention effect. In addition, these peptidebased supramolecular structures can be functionalized with targeting ligands for direct delivery of the payload to the site of interest, where they can be released. A promising new perspective appears to be the fabrication of photosensitive and photo-thermal peptide based hybrid biomaterials with antitumor activity. ${ }^{76-79}$ Although self-assembled architectures have improved stability as compared to the peptide building blocks, they lack the robustness that polymeric nanostructures offer. Therefore, peptide-polymer hybrid materials have emerged to address such problems. Peptide-polymer hybrid supramolecular assemblies can offer efficient and targeted drug and gene delivery, while stimuli-responsiveness of the polymeric component makes them interesting candidates for tissue engineering. Furthermore, spherical polymersomes with inserted peptide nanopores allow for in situ reactions within the compartments for local production and release of active molecules. Planar polymer membranes can also be used to insert peptides, and understand their functionality in synthetic templates or develop active surfaces for biosensing or ion transport. Depending on the polymer and self-assembly method chosen, each hybrid material can possess unique properties and fulfill numerous functions thus truly expanding on the versatility. 
Even though the different materials are showing promising properties, there are still some challenges to be overcome, such as control of the self-assembly process and stability of both the structure of the self-assembled peptides and stability towards degradation by proteases in vivo. Another key challenge is still the knowledge of how to obtain full and rational control of the final spatial organization of aggregating peptides at the nanoscale. ${ }^{80}$ In silico molecular modeling of self-assembling peptides can aid in overcoming this challenge but more studies are needed along with a more cost effective route to obtain large quantities of tailor made peptides. The field of peptide based biomaterials is rapidly expanding and further research is warranted for the development of such novel systems and successful translation into clinical applications.

Acknowledgements

The authors thank the University of Basel and the National Centre of Competence in Research Molecular Systems Engineering (NCCR MSE) and the Swiss National Science Foundation (SNSF) for their support. They also thank Dr. Juan Liu for help in image design.

\author{
Author Information \\ * Wolfgang Meier, Wolfgang.Meier@unibas.ch, Klingelbergstrasse 80, 4056 Basel, Switzerland \\ The authors have no other relevant affiliations or financial involvement with any organization or \\ entity with a financial interest in or financial conflict with the subject matter or materials \\ discussed in the manuscript apart from those disclosed.
}

Abbreviations 
1

2

3

4

5

6

7

8

9

10

11

12

13

14

15

16

17

18

19

20

21

22

23

24

25

26

27

28

29

30

31

32

33

34

35

36

37

38

39

40

41

42

43

44

45

46

47

48

49

50

51

52

53

54

55

56

57

58

59

60

$\alpha$-HL $\quad \alpha$-hemolysin

CCPs cell penetrating peptides

CPs cyclic peptides

DET diethylenetriamine

GFP green fluorescent protein

GUVs giant unilamellar vesicle

DOX doxorubicin

EGFR epidermal growth factor receptor

gA gramicidin $\mathrm{A}$

LUVs large unilamellar vesicles

MMP metalloproteinase

NPs nanoparticles

NTs nanotubes

PASP poly(aspartic acid)

PDMS poly(dimethylsiloxane)

pDNA plasmid DNA

PMOXA poly(2-methyloxazoline) 
TSSBM tethered solid-supported bilayer membrane

\section{References}

1. Gazit, E., Self-assembled peptide nanostructures: the design of molecular building blocks and their technological utilization. Chem. Soc. Rev. 2007, 36 (8), 1263-1269.

2. Zhang, S., Fabrication of novel biomaterials through molecular self-assembly. Nat. Biotechnol. 2003, 21 (10), 1171-1178.

3. Wang, J.; Liu, K.; Xing, R.; Yan, X., Peptide self-assembly: thermodynamics and kinetics. Chem. Soc. Rev. 2016, 45 (20), 5589-5604.

4. Yuan, C.; Li, S.; Zou, Q.; Ren, Y.; Yan, X., Multiscale simulations for understanding the evolution and mechanism of hierarchical peptide self-assembly. Phys. Chem. Chem. Phys. 2017, DOI: $10.1039 / \mathrm{c} 7 \mathrm{cp} 01923 \mathrm{~h}$.

5. Mandal, D.; Nasrolahi Shirazi, A.; Parang, K., Self-assembly of peptides to nanostructures. Org. Biomol. Chem. 2014, 12 (22), 3544-3561.

6. Ulijn, R. V.; Smith, A. M., Designing peptide based nanomaterials. Chem. Soc. Rev. 2008, 37 (4), 664-675.

7. Abbas, M.; Zou, Q.; Li, S.; Yan, X., Self-Assembled Peptide- and Protein-Based Nanomaterials for Antitumor Photodynamic and Photothermal Therapy. Adv. Mater. 2017, 29 (12), 1605021

8. Sun, L.; Zheng, C.; Webster, T. J., Self-assembled peptide nanomaterials for biomedical applications: promises and pitfalls. Int. J. Nanomed. 2017, 12, 73-86. 
9. Acar, H.; Srivastava, S.; Chung, E. J.; Schnorenberg, M. R.; Barrett, J. C.; LaBelle, J. L.; Tirrell, M., Self-assembling peptide-based building blocks in medical applications. Adv. Drug Delivery Rev. 2016, 110-111, 65-69.

10. Mandal, D.; Nasrolahi Shirazi, A.; Parang, K., Self-assembly of peptides to nanostructures. Org. Biomol. Chem. 2014, 12 (22), 3544-61.

11. Koutsopoulos, S., Self-assembling peptide nanofiber hydrogels in tissue engineering and regenerative medicine: Progress, design guidelines, and applications. J. Biomed. Mater. Res., Part A 2016, 104 (4), 1002-16.

12. de la Rica, R.; Matsui, H., Applications of peptide and protein-based materials in bionanotechnology. Chem. Soc. Rev. 2010, 39 (9), 3499-3509.

13. Zelzer, M.; Ulijn, R. V., Next-generation peptide nanomaterials: molecular networks, interfaces and supramolecular functionality. Chem. Soc. Rev. 2010, 39 (9), 3351-3357.

14. Wang, H.; Feng, Z.; Xu, B., Bioinspired assembly of small molecules in cell milieu. Chem. Soc. Rev. 2017, 46 (9), 2421-2436.

15. Sigg, S. J.; Schuster, T. B.; Meier, W. P., Self-assembled Structures from Amphiphilic Peptides. Chimia 2013, 67 (12), 881-884.

16. Dehsorkhi, A.; Castelletto, V.; Hamley, I. W., Self-assembling amphiphilic peptides. J. Pept. Sci. 2014, 20 (7), 453-467.

17. Chen, Y.; Gan, H. X.; Tong, Y. W., pH-Controlled Hierarchical Self-Assembly of Peptide Amphiphile. Macromolecules 2015, 48 (8), 2647-2653.

18. Panda, J. J.; Chauhan, V. S., Short peptide based self-assembled nanostructures: implications in drug delivery and tissue engineering. Polym. Chem. 2014, 5 (15), 4431. 
19. Cui, H.; Webber, M. J.; Stupp, S. I., Self-assembly of peptide amphiphiles: from molecules to nanostructures to biomaterials. Biopolymers 2010, 94 (1), 1-18.

20. Fan, Z.; Sun, L.; Huang, Y.; Wang, Y.; Zhang, M., Bioinspired fluorescent dipeptide nanoparticles for targeted cancer cell imaging and real-time monitoring of drug release. Nat. Nanotechnol. 2016, 11 (4), 388-94.

21. de Bruyn Ouboter, D.; Schuster, T. B.; Mantion, A.; Meier, W., Hierarchical Organization of Purely Peptidic Amphiphiles into Peptide Beads. J. Phys. Chem. C 2011, 115 (30), 14583-14590.

22. Chan, K. H.; Lee, W. H.; Zhuo, S.; Ni, M., Harnessing supramolecular peptide nanotechnology in biomedical applications. Int. J. Nanomed. 2017, 12, 1171-1182.

23. de Bruyn Ouboter, D.; Schuster, T.; Shanker, V.; Heim, M.; Meier, W., Multicompartment micelle-structured peptide nanoparticles: a new biocompatible gene- and drug-delivery tool. J. Biomed. Mater. Res., Part A 2014, 102 (4), 1155-63.

24. Bawa, R.; Fung, S. Y.; Shiozaki, A.; Yang, H.; Zheng, G.; Keshavjee, S.; Liu, M., Selfassembling peptide-based nanoparticles enhance cellular delivery of the hydrophobic anticancer drug ellipticine through caveolae-dependent endocytosis. Nanomedicine 2012, 8 (5), 647-54.

25. van Eldijk, M. B.; Schoonen, L.; Cornelissen, J. J. L. M.; Nolte, R. J. M.; van Hest, J. C. M., Metal Ion-Induced Self-Assembly of a Multi-Responsive Block Copolypeptide into WellDefined Nanocapsules. Small 2016, 12 (18), 2476-2483.

26. Zhang, N.; Zhao, F.; Zou, Q.; Li, Y.; Ma, G.; Yan, X., Multitriggered Tumor-Responsive Drug Delivery Vehicles Based on Protein and Polypeptide Coassembly for Enhanced Photodynamic Tumor Ablation. Small 2016, 12 (43), 5936-5943. 
27. Zhang, H.; Fei, J.; Yan, X.; Wang, A.; Li, J., Enzyme-Responsive Release of Doxorubicin from Monodisperse Dipeptide-Based Nanocarriers for Highly Efficient Cancer Treatment In Vitro. Adv. Funct. Mater. 2015, 25 (8), 1193-1204.

28. Doll, T. A. P. F.; Dey, R.; Burkhard, P., Design and optimization of peptide nanoparticles. J. Nanobiotechnol. 2015, 13 (1), 73.

29. Liu, L.; Xu, K.; Wang, H.; Jeremy Tan, P. K.; Fan, W.; Venkatraman, S. S.; Li, L.; Yang, Y.-Y., Self-assembled cationic peptide nanoparticles as an efficient antimicrobial agent. Nat. Nanotechnol. 2009, 4 (7), 457-463.

30. Hamley, I. W., Self-assembly of amphiphilic peptides. Soft Matter 2011, 7 (9), 4122.

31. Tian, X.; Sun, F.; Zhou, X. R.; Luo, S. Z.; Chen, L., Role of peptide self-assembly in antimicrobial peptides. J. Pept. Sci. 2015, 21 (7), 530-9.

32. Wadhwani, P.; Heidenreich, N.; Podeyn, B.; Burck, J.; Ulrich, A. S., Antibiotic gold: tethering of antimicrobial peptides to gold nanoparticles maintains conformational flexibility of peptides and improves trypsin susceptibility. Biomater. Sci. 2017, 5 (4), 817-827.

33. de Bruyn Ouboter, D.; Schuster, T. B.; Sigg, S. J.; Meier, W. P., Self-assembled peptide beads used as a template for ordered gold nanoparticle superstructures. Colloids Surf., B 2013, $112,542-7$

34. Trent, A.; Marullo, R.; Lin, B.; Black, M.; Tirrell, M., Structural properties of soluble peptide amphiphile micelles. Soft Matter 2011, 7 (20), 9572.

35. Chung, E. J.; Mlinar, L. B.; Sugimoto, M. J.; Nord, K.; Roman, B. B.; Tirrell, M., In vivo biodistribution and clearance of peptide amphiphile micelles. Nanomedicine 2015, 11 (2), 47987. 
36. Sigg, S. J.; Postupalenko, V.; Duskey, J. T.; Palivan, C. G.; Meier, W., StimuliResponsive Codelivery of Oligonucleotides and Drugs by Self-Assembled Peptide Nanoparticles. Biomacromolecules 2016, 17 (3), 935-45.

37. Sigg, S. J.; Schuster, T. B.; Meier, W. P., Self-assembled structures from amphiphilic peptides. Chimia 2013, 67 (12-13), 881-884.

38. Pugliese, R.; Gelain, F., Peptidic Biomaterials: From Self-Assembling to Regenerative Medicine. Trends Biotechnol. 2017, 35 (2), 145-158.

39. Pille, J.; van Lith, S. A.; van Hest, J. C.; Leenders, W. P., Self-Assembling VHH-ElastinLike Peptides for Photodynamic Nanomedicine. Biomacromolecules 2017, 1302-1310.

40. Gunkel-Grabole, G.; Sigg, S.; Lomora, M.; Lorcher, S.; Palivan, C. G.; Meier, W. P., Polymeric 3D nano-architectures for transport and delivery of therapeutically relevant biomacromolecules. Biomater. Sci. 2015, 3 (1), 25-40.

41. Eskandari, S.; Guerin, T.; Toth, I.; Stephenson, R. J., Recent advances in self-assembled peptides: Implications for targeted drug delivery and vaccine engineering. Adv. Drug Delivery Rev. 2016, 169-187.

42. Fatouros, D. G.; Lamprou, D. A.; Urquhart, A. J.; Yannopoulos, S. N.; Vizirianakis, I. S.; Zhang, S.; Koutsopoulos, S., Lipid-like self-assembling peptide nanovesicles for drug delivery. ACS Appl. Mater. Interfaces 2014, 6 (11), 8184-9.

43. Naskar, J.; Roy, S.; Joardar, A.; Das, S.; Banerjee, A., Self-assembling dipeptide-based nontoxic vesicles as carriers for drugs and other biologically important molecules. Org. Biomol. Chem. 2011, 9 (19), 6610-5. 
44. van Hell, A. J.; Costa, C. I.; Flesch, F. M.; Sutter, M.; Jiskoot, W.; Crommelin, D. J.; Hennink, W. E.; Mastrobattista, E., Self-assembly of recombinant amphiphilic oligopeptides into vesicles. Biomacromolecules 2007, 8 (9), 2753-61.

45. Koss, K. M.; Unsworth, L. D., Neural tissue engineering: Bioresponsive nanoscaffolds using engineered self-assembling peptides. Acta Biomater. 2016, 44, 2-15.

46. Cobo, I.; Li, M.; Sumerlin, B. S.; Perrier, S., Smart hybrid materials by conjugation of responsive polymers to biomacromolecules. Nat. Mater. 2015, 14 (2), 143-159.

47. Kowal, J. Ł.; Kowal, J. K.; Wu, D.; Stahlberg, H.; Palivan, C. G.; Meier, W. P., Functional surface engineering by nucleotide-modulated potassium channel insertion into polymer membranes attached to solid supports. Biomaterials 2014, 35 (26), 7286-7294.

48. Lomora, M.; Itel, F.; Dinu, I. A.; Palivan, C. G., Selective ion-permeable membranes by insertion of biopores into polymersomes. Phys. Chem. Chem. Phys. 2015, 17 (24), 15538-15546.

49. De Oliveira, H.; Thevenot, J.; Lecommandoux, S., Smart polymersomes for therapy and diagnosis: fast progress toward multifunctional biomimetic nanomedicines. Wiley Interdiscip. Rev.: Nanomed. Nanobiotechnol. 2012, 4 (5), 525-546.

50. Tang, W.; Becker, M. L., "Click" reactions: a versatile toolbox for the synthesis of peptide-conjugates. Chem. Soc. Rev. 2014, 43 (20), 7013-7039.

51. Golas, P. L.; Matyjaszewski, K., Marrying click chemistry with polymerization: expanding the scope of polymeric materials. Chem. Soc. Rev. 2010, 39 (4), 1338-1354.

52. Klok, H.-A., Peptide/Protein-Synthetic Polymer Conjugates: Quo Vadis. Macromolecules 2009, 42 (21), 7990-8000. 
53. Figueiredo, P.; Balasubramanian, V.; Shahbazi, M.-A.; Correia, A.; Wu, D.; Palivan, C. G.; Hirvonen, J. T.; Santos, H. A., Angiopep2-functionalized polymersomes for targeted doxorubicin delivery to glioblastoma cells. Int. J. Pharm. 2016, 511 (2), 794-803.

54. Palivan, C. G.; Goers, R.; Najer, A.; Zhang, X.; Car, A.; Meier, W., Bioinspired polymer vesicles and membranes for biological and medical applications. Chem. Soc. Rev. 2016, 45 (2), $377-411$.

55. Pangburn, T. O.; Bates, F. S.; Kokkoli, E., Polymersomes functionalized via "click" chemistry with the fibronectin mimetic peptides PR_b and GRGDSP for targeted delivery to cells with different levels of [small alpha]5[small beta]1 expression. Soft Matter 2012, 8 (16), 4449-4461.

56. van Dongen, S. F. M.; Verdurmen, W. P. R.; Peters, R. J. R. W.; Nolte, R. J. M.; Brock, R.; van Hest, J. C. M., Cellular Integration of an Enzyme-Loaded Polymersome Nanoreactor. Angew. Chem., Int. Ed. 2010, 49 (40), 7213-7216.

57. Sauer, M.; Haefele, T.; Graff, A.; Nardin, C.; Meier, W., Ion-carrier controlled precipitation of calcium phosphate in giant ABA triblock copolymer vesicles. Chem. Commun. 2001, (23), 2452-2453.

58. Nallani, M.; Andreasson-Ochsner, M.; Tan, C.-W. D.; Sinner, E.-K.; Wisantoso, Y.; Geifman-Shochat, S.; Hunziker, W., Proteopolymersomes: In vitro production of a membrane protein in polymersome membranes. Biointerphases 2011, 6 (4), 153-157.

59. Hemmatian, Z.; Keene, S.; Josberger, E.; Miyake, T.; Arboleda, C.; Soto-Rodríguez, J.; Baneyx, F.; Rolandi, M., Electronic control of $\mathrm{H}+$ current in a bioprotonic device with Gramicidin A and Alamethicin. Nat. Commun. 2016, 7, 12981. 
60. Lomora, M.; Garni, M.; Itel, F.; Tanner, P.; Spulber, M.; Palivan, C. G., Polymersomes with engineered ion selective permeability as stimuli-responsive nanocompartments with preserved architecture. Biomaterials 2015, 53, 406-414.

61. Bacinello, D.; Garanger, E.; Taton, D.; Tam, K. C.; Lecommandoux, S., EnzymeDegradable Self-Assembled Nanostructures from Polymer-Peptide Hybrids. Biomacromolecules 2014, 15 (5), 1882-1888.

62. Loyer, P.; Bedhouche, W.; Huang, Z. W.; Cammas-Marion, S., Degradable and biocompatible nanoparticles decorated with cyclic RGD peptide for efficient drug delivery to hepatoma cells in vitro. Int. J. Pharm. 2013, 454 (2), 727-737.

63. Witzigmann, D.; Wu, D.; Schenk, S. H.; Balasubramanian, V.; Meier, W.; Huwyler, J., Biocompatible Polymer-Peptide Hybrid-Based DNA Nanoparticles for Gene Delivery. ACS Appl. Mater. Interfaces 2015, 7 (19), 10446-10456.

64. Couet, J.; Samuel, J. D. J. S.; Kopyshev, A.; Santer, S.; Biesalski, M., Peptide-Polymer Hybrid Nanotubes. Angew. Chem., Int. Ed. 2005, 44 (21), 3297-3301.

65. Chapman, R.; Warr, G. G.; Perrier, S.; Jolliffe, K. A., Water-Soluble and pH-Responsive Polymeric Nanotubes from Cyclic Peptide Templates. Chem. - Eur. J. 2013, 19 (6), 1955-1961.

66. Danial, M.; My-Nhi Tran, C.; Young, P. G.; Perrier, S.; Jolliffe, K. A., Janus cyclic peptide-polymer nanotubes. Nat. Commun. 2013, 4, 2780.

67. Ulbricht, M., Advanced functional polymer membranes. Polymer 2006, 47 (7), 22172262.

68. Brogden, K. A., Antimicrobial peptides: pore formers or metabolic inhibitors in bacteria? Nat. Rev. Microbiol. 2005, 3 (3), 238-250. 
69. Menestrina, G.; Lazarovici, P., Front Matter. In Pore-forming Peptides and Protein Toxins, CRC Press: 2003; pp 1-315.

70. González-Pérez, A.; Stibius, K. B.; Vissing, T.; Nielsen, C. H.; Mouritsen, O. G., Biomimetic Triblock Copolymer Membrane Arrays: A Stable Template for Functional Membrane Proteins. Langmuir 2009, 25 (18), 10447-10450.

71. Haefele, T.; Kita-Tokarczyk, K.; Meier, W., Phase Behavior of Mixed Langmuir Monolayers from Amphiphilic Block Copolymers and an Antimicrobial Peptide. Langmuir 2006, $22(3), 1164-1172$.

72. Wong, D.; Jeon, T.-J.; Schmidt, J., Single molecule measurements of channel proteins incorporated into biomimetic polymer membranes. Nanotechnology 2006, 17 (15), 3710.

73. Martin, M.; Dubbs, T.; Fried, J. R., Planar Bilayer Measurements of Alamethicin and Gramicidin Reconstituted in Biomimetic Block Copolymers. Langmuir 2017, 33 (5), 1171-1179. 74. Song, L.; Hobaugh, M. R.; Shustak, C.; Cheley, S.; Bayley, H.; Gouaux, J. E., Structure of staphylococcal $\alpha$-hemolysin, a heptameric transmembrane pore. Science 1996, 274 (5294), $1859-1865$.

75. Zhang, X.; Fu, W.; Palivan, C. G.; Meier, W., Natural channel protein inserts and functions in a completely artificial, solid-supported bilayer membrane. Sci. rep. 2013, 3, 1-7.

76. Liu, K.; Xing, R.; Zou, Q.; Ma, G.; Möhwald, H.; Yan, X., Simple Peptide-Tuned SelfAssembly of Photosensitizers towards Anticancer Photodynamic Therapy. Angew. Chem., Int. Ed. 2016, 55 (9), 3036-3039.

77. Zou, Q.; Abbas, M.; Zhao, L.; Li, S.; Shen, G.; Yan, X., Biological Photothermal Nanodots Based on Self-Assembly of Peptide-Porphyrin Conjugates for Antitumor Therapy. $J$. Am. Chem. Soc. 2017, 139 (5), 1921-1927. 
78. Liu, K.; Xing, R.; Li, Y.; Zou, Q.; Möhwald, H.; Yan, X., Mimicking Primitive Photobacteria: Sustainable Hydrogen Evolution Based on Peptide-Porphyrin Co-Assemblies with a Self-Mineralized Reaction Center. Angew. Chem., Int. Ed. 2016, 55 (40), 12503-12507. 79. Liu, K.; Xing, R.; Chen, C.; Shen, G.; Yan, L.; Zou, Q.; Ma, G.; Möhwald, H.; Yan, X., Peptide-Induced Hierarchical Long-Range Order and Photocatalytic Activity of Porphyrin Assemblies. Angew. Chem., Int. Ed. 2015, 54 (2), 500-505.

80. Colombo, G.; Soto, P.; Gazit, E., Peptide self-assembly at the nanoscale: a challenging target for computational and experimental biotechnology. Trends Biotechnol. 2007, 25 (5), 211 218.

TOC Image 


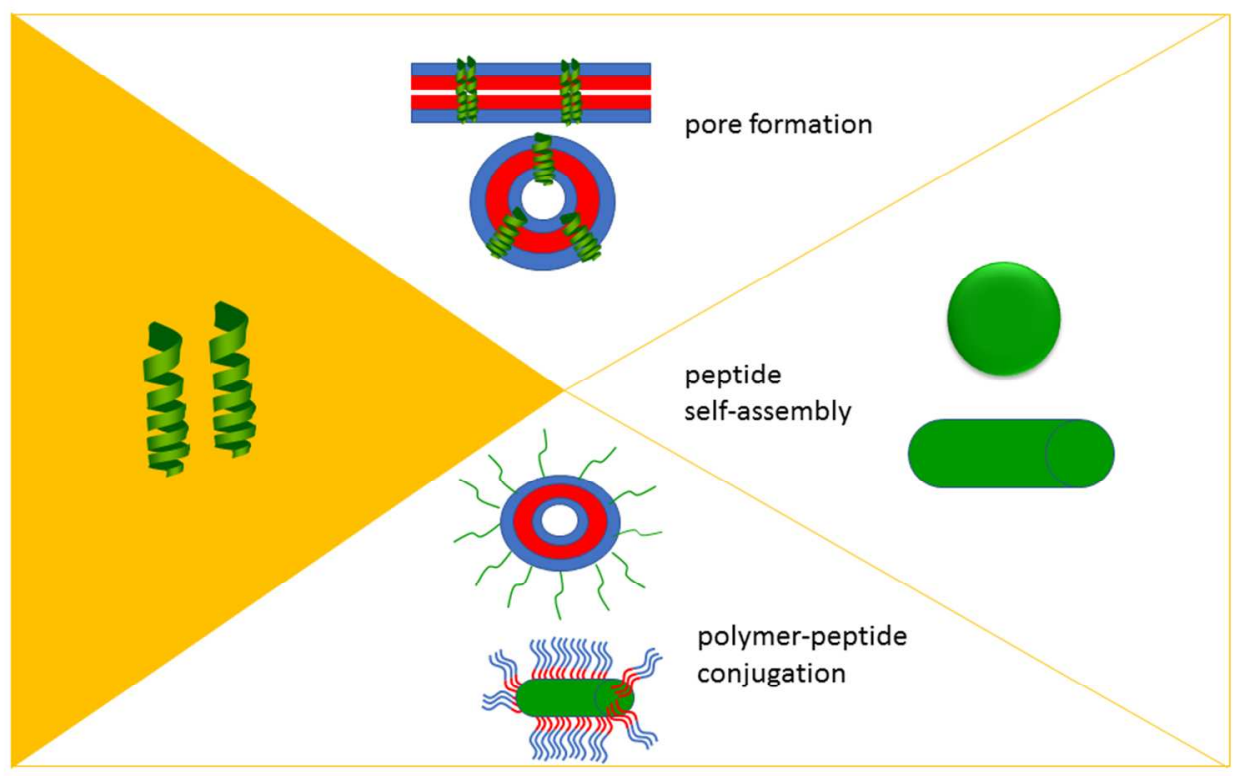



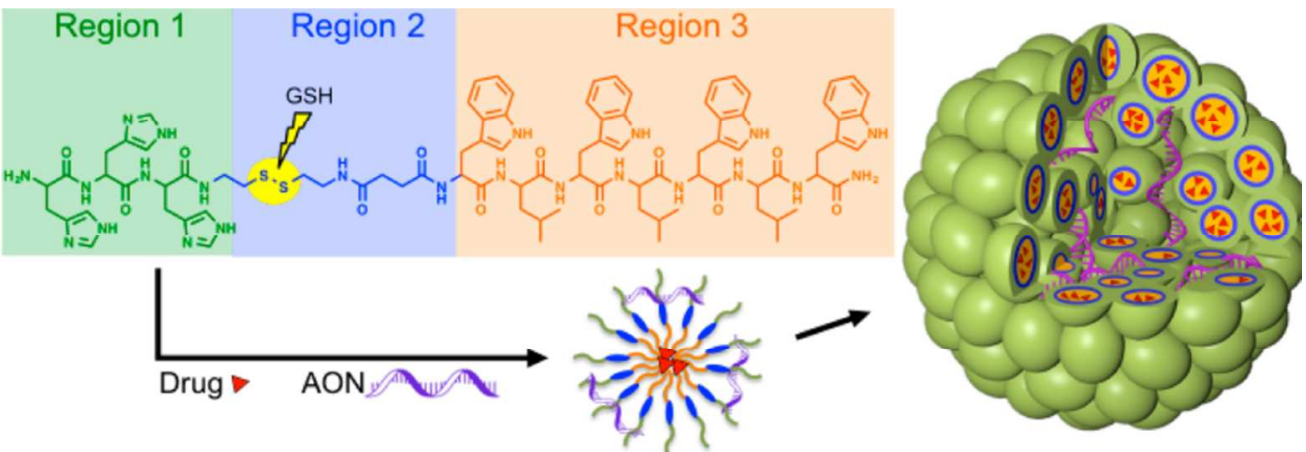

Self-assembly of amphiphilic peptides containing a histidine rich hydrophilic domain (region 1) connected by reduction sensitive linker (region 2) to a tryptophan rich hydrophobic domain (region 3). A two-step selfassembly can occur: first, the peptides assemble into micelles loaded with DOX within the inner hydrophobic core and a nucleotide in the hydrophilic exterior; secondly, the micelles cluster together to form multicompartment micelles

$139 \times 48 \mathrm{~mm}(220 \times 220 \mathrm{DPI})$ 


\section{Encapsulation of DOX into peptide-polymer vesicles, functionalized with peptide Angiopep2. Reprinted from "Angiopep2-functionalized polymersomes for targeted DOX delivery to glioblastoma cells"}

\section{$164 \times 65 \mathrm{~mm}(220 \times 220$ DPI $)$}


CLSM images of a giant unilamellar vesicle (GUV) composed of A7B49A7 triblock copolymer in the presence of: (A) Bodipy (red), (B) labeled gA-OG488 (green), (C) Overlay of A and B (red and green). Fluorescent intensity change over time of 5(6)-carboxyfluorescein inside and outside of polymer GUVs (D) in absence and $(E)$ after insertion of $\mathrm{gA}$

\section{$338 \times 190 \mathrm{~mm}(96 \times 96 \mathrm{DPI})$}


. CP-polymer NTs with two corona configurations: (A) Janus assembly with 'demixed' corona. (B) Hybrid assembly with 'mixed' corona (C) Calcein dye leakage experiment using LUVs with $2 \mu \mathrm{M}$ Janus conjugate. No dye leakage was observed for non-Janus conjugate

$338 \times 190 \mathrm{~mm}(96 \times 96$ DPI $)$ 

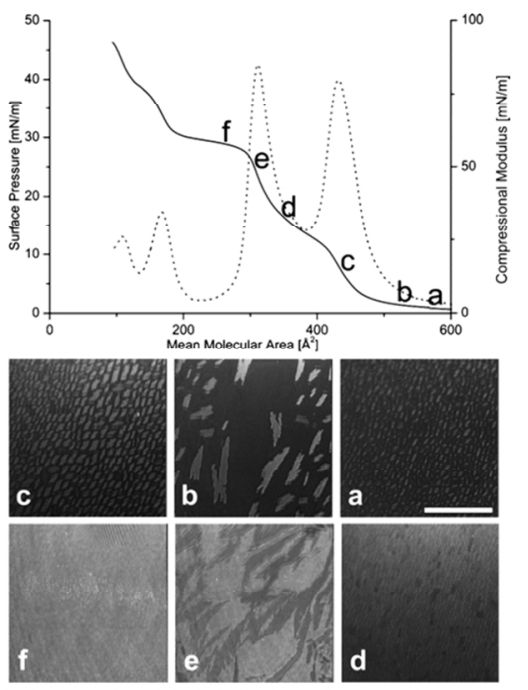

BAM images of a PMOXA13PDMS23PMOXA13-alamethicin (molar ratio 0.3:0.7) monolayer at different surface pressures.

$338 \times 190 \mathrm{~mm}(96 \times 96 \mathrm{DPI})$ 


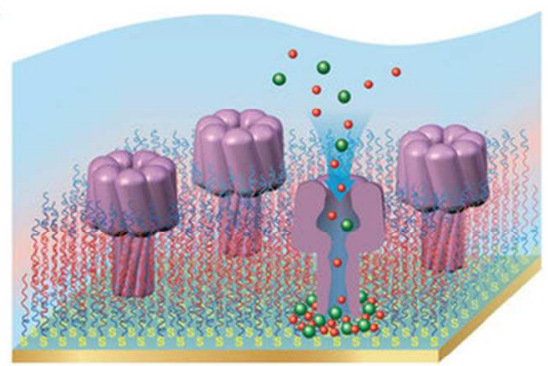

B

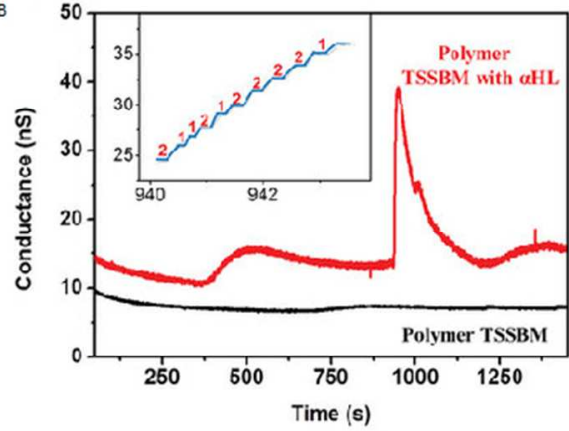

Schematic representation of the TSSBM (A) and conductance measurement across it (B).

$223 \times 84 \mathrm{~mm}(96 \times 96 \mathrm{DPI})$

3

4

6

9

10

11

12

14

15

16

17

20

21

22

23

24

25

26

27

28

29

30

31

32

33

34

35

36

37

38

39

40

41

42

43

44

45

46

47

48

49

50

51

52

53

54

55

56

57

58

59

60 


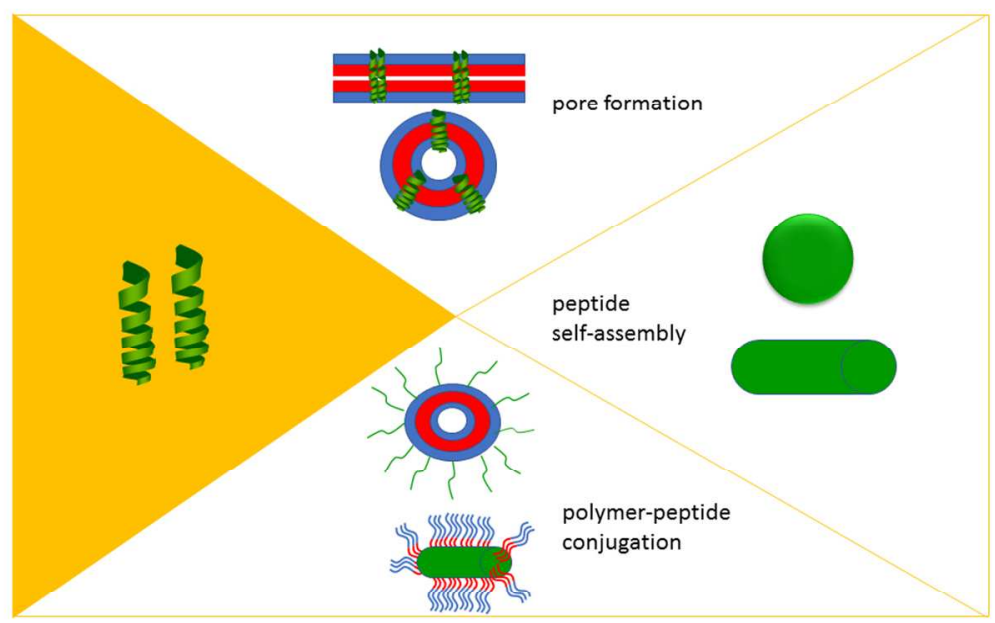

TOC

$338 \times 190 \mathrm{~mm}(96 \times 96$ DPI) 